\title{
Experience with telemedicine in paediatric nephrology during the COVID pandemic
}

\author{
Sanjeev Gulati ${ }^{1}$ (i) $\cdot$ Amrita Sengar ${ }^{1}$
}

Received: 29 March 2021 / Revised: 4 April 2021 / Accepted: 8 April 2021 / Published online: 19 May 2021

(C) IPNA 2021

To the Editor,

We read with great interest the article by Tse et al. about the problems faced by children and young adults with kidney disease during the lockdown [1]. In such a scenario, telemedicine is a useful tool in mitigating the issues as well as providing a modality for continued care. Telemedicine is the use of electronic methods to deliver health care and/or health education from a distance [2]. It has been used to deliver health care services in countries like the USA and Australia which have sparse populations spread over large geographically isolated areas [3]. The unprecedented lockdown created further logistical barriers for children with kidney diseases, as there are very few trained paediatric nephrologists in the country and the majority are based in cities. This single-centre prospective pilot study, the first of its kind during the pandemic, was conducted to evaluate the feasibility and applicability of telemedicine services in the management of children with kidney diseases. The objective was to provide consultation for younger as well as older children with kidney diseases because of restricted access in view of the lockdown imposed all over the country in the wake of the COVID pandemic. Another objective was to prioritise and select children who needed inpatient care. The study was conducted during the national lockdown in India between 23 March and 30 June 2020. The patients were informed about the availability of teleconsultation services through SMS (short message service) messages using our patient database. To ensure wider reach, we also used Facebook and WhatsApp to send these messages. The medical data received was

Sanjeev Gulati

sgulati2002@gmail.com

Fortis FLT LT Rajan Dhall Hospital, Delhi, New Delhi, India entered in the Healthplix electronic medical records (EMR) system. On receiving the request for a telenephrology consult from the child's parents/caregivers, an SMS was sent requesting information about data including age, weight, height and vital signs including blood pressure (where required) as well as current and previous investigations. The information was entered into EMR and a prescription was emailed or sent via WhatsApp as per family preference. At the end of the e-consult, the parents/caregivers were asked to rate the teleconsultation experience on a scale of 0 (not satisfied) to 10 (fully satisfied). The study was approved by the institutional ethics committee.

During the study period, a total of 178 e-consults were done. Of these, 174 consults were for domestic patients and 4 were for international patients. The study group comprised 90 patients - 59 boys and 31 girls. All of the patients were provided teleconsultation using a combination of a WhatsApp text and a phone call. The distribution of the diagnoses was as follows: idiopathic nephrotic syndrome (53), chronic kidney disease (17), kidney transplant (7), UTI (5), acute glomerulonephritis (4), acute kidney injury (2) and other (2). The mean age of the children in this study was 9 years (range $0.17-18$ years). Of the 178 e-consults that were advised, all opted for teleconsultation. Of the 90 children in the study, 10 were new patients who were evaluated for the first time via teleconsultation. The other 80 children were follow-up patients in our OPD clinics. Of the 90 children who were given e-consults, 87 were advised follow-up e-consults and 3 were advised admission. We found this to be an effective modality in triaging children with kidney diseases for follow-up or admission. Based on teleconsultation, $3 / 90$ (3.4\%) of the children were successfully triaged into admission. This avoided the need for OPD visits in the other $96.6 \%$ who were managed by teleconsultations, sparing them the logistical nightmare of travel to the hospital for 
specialised paediatric nephrology review. The patient/ family satisfaction score for e-consults was 9.7. Telemedicine has been found to improve clinical outcomes due to better compliance as well as easy and better follow-up. This results in enhanced satisfaction due to a stronger doctor-patient relationship and improved clinical outcomes. Telemedicine can be used by paediatric nephrologists for new patients, follow-up patients, second opinions and cross referrals.

Based on our experience, we conclude that telenephrology offered an effective method for providing paediatric nephrology services during the period of pandemic lockdown, when access to paediatric nephrology care was limited. It is also an effective modality in providing individualised tailored advice to this vulnerable segment of the population.

\section{References}

1. Tse Y, Darlington ASE, Tyerman K, Wallace D et al (2021) COVID19: experiences of lockdown and support needs in children and young adults with kidney conditions. Pediatr Nephrol. https://doi. org/10.1007/s00467-021-05041-8

2. WHO Global Observatory for eHealth (2010) Telemedicine: opportunities and developments in Member States: report on the second global survey on eHealth. World Health Organization. https://apps. who.int/iris/handle/10665/44497

3. Trnka P, White MM, Renton WD, McTaggart SJ, Burke JR, Smith AC (2015) A retrospective review of telehealth services for children referred to a paediatric nephrologist. BMC Nephrol 16:1-7

Publisher's note Springer Nature remains neutral with regard to jurisdictional claims in published maps and institutional affiliations. 